\title{
Bioanalysis - but not as we knew it: an AstraZeneca perspective of the last 10 years evolution to meet a diversifying portfolio
}

\author{
Glen Hawthorne ${ }^{1}$, Neil Henderson ${ }^{1}$, Mikko Hölttä ${ }^{1}$, Craig Stovold ${ }^{1}$, Åsa Wåhlander ${ }^{1}$ \& \\ Amanda Wilson*,1 \\ ${ }^{1}$ Translational Biomarkers \& Bioanalysis, Drug Safety \& Metabolism, IMED Biotech Unit, AstraZeneca, Cambridge, UK \\ *Author for correspondence: amanda.wilson@astrazeneca.com
}

\begin{abstract}
' 6 there has been a major transformation within the AstraZeneca bioanalytical laboratory, seeing the small molecule-centric LC-MS/MS capability being reduced and replaced with a diverse suite of technologies suitable to meet the new modality requirements."
\end{abstract}

First draft submitted: 30 January 2019; Accepted for publication: 21 February 2019; Published online: 18 April 2019

Keywords: advanced medicines • antisense oligonucleotides (ASOs) • CRISPR-Cas9 • messenger RNA (mRNA) • nanomedicine $\bullet$ outsourcing $\bullet$ peptides $\bullet$ portfolio $\bullet$ protein

AstraZeneca is committed to driving scientific advances in small molecule, nucleotide and other emerging technology platforms and over the last decade, there have been many exciting breakthroughs delivering novel therapeutics. A better understanding of underlying disease biology to develop more targeted approaches is providing an opportunity to deliver specialty medicines for disease mechanisms that were previously considered difficult to target [1]. AstraZeneca is shifting away from developing standard small-molecule treatments and are progressively tailoring the pipeline to include sophisticated drug-delivery initiatives and now greater than $30 \%$ of the drug programs explore new modalities. The diversified portfolio has driven an exciting evolution in the bioanalysis laboratory environment and the analytical challenges have changed to align with the nature of the drug pipeline. Historically, the in-house capability was focused upon small molecule analysis using HPLC coupled with MS, and now to meet the requirements of the greater molecular diversity, the bioanalysis laboratory has needed to review its capabilities and increase the technical flexibility of our scientists to access different methodologies interchangeably to meet the changing analytical demands. As such the bioanalytical operating model has been assessed iteratively in recent years to align with the portfolio transformation, including $100 \%$ outsourcing of routine small molecule bioanalysis support for toxicology evaluation, enabling the internal capability to concentrate on scientific innovation. The new challenges have necessitated the integration of novel strategic approaches with innovative application of traditional analytical tools to enable bioanalytical support to drug discovery and development. Here we will share an insight to the Translational Biomarker and Bioanalysis group (TBB) analytical strategies used to support AstraZeneca's new treatment approaches including antisense oligonucleotides (ASOs) and modified messenger RNA (mRNA) therapeutics that modulate protein production in vivo, novel protein therapeutics and novel approaches to small-molecule drug delivery.

\section{Antisense oligonucleotides}

Antisense oligonucleotides function by regulating the translation of mRNA into protein, and thereby reducing the amount of target protein expressed [2]. ASOs are typically rapidly cleared from plasma by uptake into mainly kidney and liver, therefore one of the key challenges associated with these molecules is achieving adequate exposure in other target tissues. Exposure in on- and off-target tissues drives an increased need for the understanding of the quantitative biodistribution of the ASO, not just in terms of the therapeutic but also in terms of the efficacy: is the ASO that is present in any tissue having a local or holistic therapeutic effect? 
As an approach to improve therapeutic windows, AstraZeneca is exploring a range of options for targeting an ASO to a specific site-of-action. These range from 'simple' GalNAc-ASO conjugates to improve liver exposure, to more complex peptide-ASO conjugates intended to drive cellular uptake to more specific subpopulations of cells [3]. These more complicated molecules combining two physicochemically differing entities can require more extensive bioanalytical input - measurement of both the ASO fragment and the peptide conjugate (and in some cases the Linker molecule). Current generation ASOs are very stable analytes with similar separation characteristics, mainly dictated by the length of the strand. Multiple technologies are utilized; in-house we use highly specific LC-MS/MS detection based upon the phosphorothioate backbone (an entity not present naturally), which avoids any interference from endogenous oligonucleotides, or high sensitivity hybridization assays (e.g., HELISA) with minimal binding of truncated forms can be applied; whereas we have leveraged the skills of CROs to deliver HPLCUV routinely - a sensitive method that is also able to quantify metabolites, unlike HELISA. Method approach is influenced by sample number, expected analyte concentrations and of course project question.

For PD assessment of the ASO, real-time quantitative PCR (RT-qPCR) is the preferred choice to determine target gene knock down. For the mRNA knockdown analysis, choice of appropriate reference mRNA and selection of a tissue expressing a relevant level of the target mRNA enables the production of reliable data.

\section{Novel protein therapeutics}

Large-molecule therapeutics, such as antibodies, have been major players in drug portfolios for some time now; however, there are now numerous novel protein entities emerging. The current AstraZeneca portfolio includes modified proteins, peptides and recombinant enzymes. To support the development of protein therapeutics, AstraZeneca employs both immunoassay- and MS-based analytical techniques to provide an integrated assessment of PK, PD and immunogenicity.

We have found that a close relationship is required between the scientists performing the PK, PD and immunogenicity assays to be able to better understand and interpret exposure of the active therapeutic. In order to demonstrate that the therapeutic is active for the duration of a study we deploy a mix of 'free' and 'total' PK assays alongside a PD end point, such as enzyme activity or receptor activation assay. The need to develop and use an immunogenicity assay in nonclinical studies is based upon a combination of scientific evidence and clinical immunogenicity risk. Selection of the right assay platform can be critical in ensuring the measurement of the desired end point, with LC-MS and ligand-binding assays viewed as complimentary techniques providing different insights. We focus on low microliter volume methodologies to enable delivery of multiple end points from a single subject sample, and steer assay development needs to include an understanding of interferences from drug target and anti-drug antibodies.

\section{Modified mRNA}

Through the administration of modified messenger RNA (mRNA) drugs, we can utilize the body's own cellular machinery to 'fine tune' the production of an efficacious protein in vivo. Modifications to the nucleotide bases (and sequences) are designed to enhance both PK and PD properties. Even with modifications, which confer increased stability, modified mRNA can be highly susceptible to degradation by nuclease enzymes present in the blood and so may need to be administered (as cargo) in protective vesicles, such as lipid nanoparticles (LNPs).

In fact, to fully understand the safety and PK/PD relationship for a modified mRNA drug project, numerous bioanalytical end points may be required, including analysis of the mRNA, the produced protein, the LNP and markers of immune activation in response to 1-3 (including anti-drug antibodies and inflammatory cytokine profiles). It is also important to consider the potential holistic safety impact of these drug components through biodistribution assessments and quantitative biodistribution in target safety and efficacy tissue is common place in mRNA projects. To further complicate the bioanalytical challenge, the different analytes can have noncompatible sample processing procedures, which may have consequences on the study design in order to generate sufficient analyzable matrix. In fact, a key component of any study setup is ensuring that optimal sample collection occurs, for example, whole blood is collected directly into Paxgene protectorate for mRNA analysis or plasma is acidified prior to freezing in order to stabilize the LNP component; these steps are essential to have confidence in the data generated and the decisions made upon that data.

In the Discovery setting, the assay choice for the measurement of the derived protein is often driven by the immunoassay reagent or commercial kit availability and whether it is possible to achieve adequate sensitivity for the project's needs. In the absence of suitably specific antibodies, the default approach is LC-MS/MS. Good 
communication and early engagement with a project team has proven essential if there is a need to produce custom reagents in-house, albeit good antibodies can typically take 6 months to generate. For analysis of the mRNA, the primary approach used in-house is the branched DNA methodology. This commercially available method has the advantage of requiring no sample purification, as opposed to real-time qPCR, which theoretically has the potential to aid in higher bioanalytical throughput. As with the administration of novel therapeutic (human) proteins, the bioanalyst may need to consider development of ADA screening assays for the modified mRNA-encoded protein in preclinical species to help in the interpretation of PK/PD responses over time.

The long list of potential analytical end points available to modified mRNA project teams can be a challenge to the bioanalytical laboratory due to impact of the resource required, both in terms of consumables and FTEs. Again, we have found that a greater interaction between the bioanalytical scientist and project team, discussing the biology as well as the bioanalytical limitations of a study, can highlight the real necessity to prioritize the 'need to know' rather than 'nice to know' questions to progress a project. As with many of the other new modalities, however, most questions for these drug projects are in the 'need to know' category and so management of resource is just as important as application of technical innovation.

\section{CRISPR-Cas9 gene editing}

During recent years gene-editing technology in the form of CRISPR-Cas9 has been used extensively for precise genome editing in vitro and in preclinical studies [4,5] and has great potential as a therapeutic [6]. Recently, groups have started to explore the use of gene editing by CRISPR-Cas9 for use in clinical studies to target/treat patients with medical conditions such as sickle cell disease and tumors (many listed on clinicaltrials.gov [7]). From a bioanalytical perspective, there are to date no regulatory guidelines for what needs to be analyzed from the Cas 9 complex. The complex is constituted by the Cas9 protein that has a guide RNA (gRNA) bound to it which targets the complex to the correct sequence in the DNA to introduce a cut or break, deletion or substitution. The primary readout for gene-editing experiments have been gene knockdown and editing efficiency; the exposure of the Cas 9 protein and the gRNA, are not usually explored (or at least not reported). There are currently no quantitative, qualified methods reported for measuring the constituents of the Cas 9 complex. Our focus has been on developing quantitative assays for Cas9 to measure in in vitro and in vivo experiments to understand the relationship between the Cas 9 exposure versus on-target gene editing efficiency in relationship to off-target effects. The ability to quantify circulating Cas9, which theoretically could have the potential to cause off target editing in unwanted tissues has also been a driver for quantitative assays. A variety of different analytical approaches/tools will be required to analyze the Cas 9 complex as a whole or as the individual components; immunoassays, PCR, hybridization platforms/assays can be used to understand the exposure of the respective parts as well as determining the ratio between the Cas9 protein and gRNA. A further relevant approach might be using an activity-based assay to show whether the Cas 9 complex is active or not.

\section{Novel delivery of small molecules}

Over the past 10 years, the percentage of small-molecule projects in the AstraZeneca portfolio has decreased. However, small molecules continue to play an important role in the AstraZeneca pipeline, especially compounds for receptor targets, which would previously not have been considered for development, due to poor physiochemical properties or ADME properties. Through the clever application of nanomedicines, such as dendrimers and other nanoformulations, the 'druggability' of these small molecules has become possible.

However, these approaches have increased the complexity for bioanalysts, since end points are required for circulating 'total' drug to understand the longevity of pharmacological effect as well as a released circulating drug concentration, which drives the potential toxicological and therapeutic thresholds. Since the amount of therapeutic agent contained within a nanomedicine can be very large relative to the circulating released drug the handling of the samples is critical to not generate artificially high levels of released drug through ex vivo degradation of the nanomedicine. Stabilization approaches are investigated during method development and even with best efforts it is not always possible to fully control the sample handling procedures and a fit-for-purpose approach may be required. Often blood or plasma samples are split on collection, one for a 'total' measurement and the other for 'released' measurement to minimize the sample manipulation, however, splitting samples reduce the available matrix volume for analysis, requiring sensitive assays to be developed using low sample volumes.

In addition to stability and sensitivity considerations for assays of nanomedicines, the availability of wellcharacterized reference materials is essential for monitoring the quality of these complex assays appropriately where 
a combination of free analytical reference standard and nanomedicine is required. Draft industry guidelines exist to harmonize the requirements for the development of this rapidly evolving class of therapeutics [8].

\section{Bioanalysts answering a diverse set of questions}

It is not just the expansion of drug modality types that has an impact upon the way AstraZeneca has approached bioanalysis. A major influence on the AstraZeneca bioanalysis laboratory, and indeed the rest of the company, over the past 10 years is the implementation of the 5R framework. An excerpt from the 2018 paper [9] highlights this renewed focus:

"In 2011, AstraZeneca began a major revision of its RéD strategy with the aim of improving its R\& $D$
productivity, led to the development and implementation of the '5R framework', which is focused on five
technical determinants (the right target, right tissue, right safety, right patient and right commercial potential).
AstraZeneca scientists now work in fewer disease areas, with a focus on a deeper scientific understanding of
disease biology and mechanisms. The company has also made substantial investments in its capabilities for target
selection and validation, lead generation, PK/PD modeling, patient stratification and biomarkers."

This change in mindset has helped shape the portfolio to what it is today, and with it the role of the bioanalytical scientist in delivering data for preclinical safety evaluation has evolved along with the portfolio. Study designs are being maximized in order to answer drug metabolism, PK, PD and where appropriate immunogenicity questions that allow a fully integrated drug safety and metabolism evaluation. The resource requirements of advanced medicines are certainly not substitutional for the traditional small molecule bioanalysis and toxicokinetic evaluation. For these novel modalities, multiple bioanalytical arms are incorporated into toxicology studies and various matrices are often evaluated to develop an initial understanding of drug biodistribution using quantitative tissue analysis. In regulatory facing toxicology studies, drug exposure evaluation in principal matrices are supported using fully validated methods (at CRO partners) and secondary tissue drug measurements are generated using appropriately characterized methods.

In all, the diverse questions asked by the company in support of these modalities have led the TBB group to ensure a mix of immunoassay, MS and molecular biology expertise at one or both geographical locations.

\section{Outsourcing: collaboration is key}

Building good relationships between laboratories has always formed a key element of AstraZeneca's bioanalytical strategies, but even more so for the support of novel therapeutics over the last 10 years. Where drug candidates are in-licensed, the transfer of challenging methods between laboratories at the originator (early discovery), AstraZeneca (investigational toxicology, PK and PK/PD) and CROs (GLP and GCP studies) requires close collaboration. An open relationship between all parties allows us to capitalize on the experiences of each facility and share best practices and learning points. In the selection of preferred partners for the support of our advanced medicines portfolio, we have looked toward shared values, scientific focus and broad analytical capabilities in an effort to complement our in-house capability or provide a niche service for PK/PD and immunogenicity analysis.

For many of the analytical techniques now used in the support of advanced medicines, critical reagent management and good bridging strategies are also key challenges to overcome in the provision of consistent bioanalytical methods. Reagents for the measurement of novel drug substances may have been developed by partners or commercial suppliers and so careful monitoring of use should be maintained at each facility to ensure all contributing laboratories will have sufficient future access to materials. In the field of critical reagent development, it is also prudent to understand the potential use of a reagent at the outset to ensure production and characterization can be conducted to meet the quality needs of all the end users. In our experience, and as you transition from discovery to development, it is important to have a clear strategy for long-term reagent provision and define the accountabilities for delivery of appropriate amounts of appropriately characterized materials. For these reasons, communication and engagement across numerous parties and a highly collaborative mindset continues, and will continue, to be a key factor in progressing drug projects, novel modalities or otherwise.

\section{Summary}

During the last 10 years, there has been great change in the medicines pipeline, which has significantly impacted the status quo within the bioanalytical arena. More recently there has been a major transformation within the AstraZeneca bioanalytical laboratory, seeing the small molecule-centric LC-MS/MS capability being reduced and 
replaced with a diverse suite of technologies suitable to meet the new modality requirements. Technical creativity to answer biological, as well as bioanalytical, questions has become key to successful delivery of a novel and changing portfolio. Building and integrating diverse skills and experience within AstraZeneca and partners is contributing toward this success; and with newer molecules on the portfolio horizon, including PROTACs and the expanding use of novel delivery systems, and application of advanced computer systems to streamline processes, we predict the next 10 years will still be full of interesting strategies and challenges for the bioanalyst.

\section{Financial \& competing interests disclosure}

Authors are employees of AstraZeneca and all hold AstraZeneca shares. The authors have no other relevant affiliations or financial involvement with any organization or entity with a financial interest in or financial conflict with the subject matter or materials discussed in the manuscript apart from those disclosed.

No writing assistance was utilized in the production of this manuscript.

\section{References}

1. Valeur E, Guéret SM, Adihou H et al. New modalities for challenging targets in drug discovery. Angew. Chem. Int. Ed. Engl. 56(35), 10294-10323 (2017).

2. Andersson S, Antonsson M, Elebring M, Jansson-Lofmark R, Weidolf L. Metabolism and pharmacokinetic strategies for oligonucleotide- and mRNA-based drug development. Drug Discov. Today 23(10), 1733-1745 (2018).

3. Ämmälä C, Drury WJ 3rd, Knerr L et al. Targeted delivery of antisense oligonucleotides to pancreatic $\beta$-cells. Sci. Adv. $4(10)$, eaat3386 (2018).

4. Sander JD, Joung JK. CRISPR-Cas systems for editing, regulating and targeting genomes. Nat. Biotechnol. 32(4), 347-355 (2014).

5. Fellmann C, Gowen BG, Lin PC, Doudna JA, Corn JE. Cornerstones of CRISPR-Cas in drug discovery and therapy. Nat. Rev. Drug Discov. 16, 89-100 (2017).

6. Koo T, Kim JS. Therapeutic applications of CRISPR RNA-guided genome editing. Brief. Funct. Genomics 16, 38-45 (2017).

7. ClinicalTrials.gov

8. Drug Products, Including Biological Products, that Contain Nanomaterials: Guidance for Industry (December 2017). www.fda.gov/downloads/Drugs/GuidanceComplianceRegulatoryInformation/Guidances/UCM588857.pdf

9. Morgan P, Brown DG, Lennard S. Impact of a five-dimensional framework on R\&D productivity at AstraZeneca. Nat. Rev. Drug Discov. 17(3), 167-181 (2018). 
